\title{
ARTICLE
}

\section{Horizontal gene transfer converts non-toxigenic Clostridium difficile strains into toxin producers}

\author{
Michael S.M. Brouwer ${ }^{1}$, Adam P. Roberts ${ }^{1}$, Haitham Hussain ${ }^{1}$, Rachel J. Williams ${ }^{1}$,
} Elaine Allan ${ }^{1} \&$ Peter Mullany ${ }^{1}$

Clostridium difficile is a major nosocomial pathogen and the main causative agent of antibioticassociated diarrhoea. The organism produces two potent toxins, $A$ and $B$, which are its major virulence factors. These are chromosomally encoded on a region termed the pathogenicity locus (PaLoc), which also contains regulatory genes, and is absent in non-toxigenic strains. Here we show that the PaLoc can be transferred from the toxin-producing strain, 630 $\Delta$ erm, to three non-toxigenic strains of different ribotypes. One of the transconjugants is shown by cytotoxicity assay to produce toxin $B$ at a similar level to the donor strain, demonstrating that a toxigenic $C$. difficile strain is capable of converting a non-toxigenic strain to a toxin producer by horizontal gene transfer. This has implications for the treatment of $C$. difficile infections, as non-toxigenic strains are being tested as treatments in clinical trials.

\footnotetext{
${ }^{1}$ Department of Microbial Diseases, UCL Eastman Dental Institute, University College London, 256 Gray's Inn Road, London WC1X 8LD, UK. Correspondence and requests for materials should be addressed to P.M. (email: p.mullany@ucl.ac.uk).
} 
C lostridium difficile-associated diarrhoea (CDAD) can range from mild to severe and can be fatal due to complications such as pseudomembranous colitis and toxic megacolon ${ }^{1}$. CDAD is almost always associated with antibiotic usage, which disrupts the normal gut microflora allowing $C$. difficile colonization ${ }^{2}$. The organism produces two potent toxins, $\mathrm{A}$ and $\mathrm{B}$, which disrupt the gut epithelium and are the major virulence factors of this organism 3,4 . Both toxins are encoded on the pathogenicity locus (PaLoc), a 19.6-kb chromosomal region that also encodes potential transcriptional regulators and a holin-like protein. The entire PaLoc is absent from non-toxigenic strains replaced by a highly conserved $115 \mathrm{bp}$ non-coding region at this genomic site ${ }^{5}$.

C. difficile $630 \Delta \mathrm{erm}^{6}$ is an erythromycin-sensitive derivative of the toxigenic C. difficile 630 (ref. 7) and contains the PaLoc ${ }^{3,4}$. This strain also contains at least six active conjugative transposons (CTns), genetic elements that are generally integrated into the genome but are capable of mediating their own transfer to suitable recipients ${ }^{8}$. Conjugative transfer was shown for CTn1, CTn2, CTn4, CTn5, CTn7 and Tn5397, all of which can be transferred to the non-toxigenic $C$. difficile strain CD37 (refs 9-11). The aim of the current investigation was to determine if any other genetic information is cotransferred with the CTns. We demonstrate that the PaLoc can be transferred to CD37, which results in this non-toxigenic strain being converted to a toxin producer.

\section{Results}

The PaLoc transfers to non-toxigenic C. difficile CD37. To determine whether genetic elements were cotransferred in the absence of direct selection when transfer of CTn 1 was selected, we initially investigated nine CD37 transconjugants containing CTn1::ermB (an erythromycin-resistant derivative of CTn1 (ref. 9)) by PCR and subsequent DNA sequencing (see Methods section for details). One of these strains had unexpectedly acquired the PaLoc. To determine whether the PaLoc could

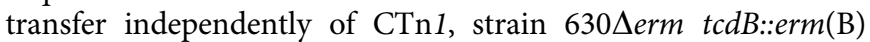
(Table 1$)^{4}$ containing a genetically marked PaLoc was used as a donor with CD37 as the recipient. PaLoc-containing transconjugants were obtained at a frequency of $7.5 \times 10^{-9}$ transconjugants per donor $\left(\right.$ s.d. $\left.=4.2 \times 10^{-9}\right)$, comparable to transfer frequencies previously reported for other CTns that are transferred between these strains 9 .

It is important to determine whether the newly acquired toxin genes are capable of directing toxin production. To do this, one of the PaLoc-containing transconjugants that had acquired the wildtype PaLoc (that is, one of the transconjugants that contained CTn1 and the PaLoc) was selected for further study and designated PaLoc386 (Table 1). This strain was subject to an in vitro cytotoxicity assay with HFF-1 cells. Filter-sterilized cell supernatants were incubated with a monolayer of HFF-1 cells for $24 \mathrm{~h}$ before the cytopathic effect (CPE) was determined (Fig. 1a). The end point toxin titre ${ }^{4}$ was determined for $630 \Delta \mathrm{erm}$, PaLoc386 and CD37 (Fig. 1c). PaLoc386 produced similar levels of toxin to the donor strain $630 \Delta \mathrm{erm}$, whereas supernatant from the recipient strain $\mathrm{CD} 37$ was negative for toxin production (Fig. 1c). Addition of a commercial TcdB antiserum (see Methods) abolished the CPE of both the 630 4 erm and PaLoc386 supernatants indicating that the two strains produced functional toxin B (Fig. 1c).

The PaLoc is transferred on variable-sized DNA fragments. To investigate the mechanism underlying the PaLoc transfer, wholegenome sequences were determined for seven transconjugants containing either the marked or wild-type PaLoc. The sequence of
Table 1 | Bacterial strains used in this study.

\begin{tabular}{|c|c|c|}
\hline Strain (Ribotype) & Properties & $\begin{array}{l}\text { Reference } \\
\text { or source }\end{array}$ \\
\hline $630(012)$ & $\mathrm{Tc}^{\mathrm{R}} \mathrm{Erm}^{\mathrm{R}} \mathrm{Rif}^{\mathrm{S}}$ & 26 \\
\hline $630 \Delta$ erm (012) & $\mathrm{Tc}^{\mathrm{R}} \mathrm{Erm}^{\mathrm{S}} \mathrm{Rif}^{\mathrm{S}}$ & 6 \\
\hline CD37 (009) & $\mathrm{TC}^{\mathrm{S}} \mathrm{Erm}^{\mathrm{S}}$ Rif $^{\mathrm{R}}$ non-toxigenic & 27 \\
\hline CD843 & $\begin{array}{l}\text { Positive control for } \varphi \text { C630-1 and } \\
\varphi \mathrm{C} 630-2\end{array}$ & 28 \\
\hline $\begin{array}{l}\text { 630 Lerm CTn1 } \\
\text { CD0386::erm(B) } \\
\text { (012) }\end{array}$ & $\begin{array}{l}\text { ClosTron mutant, donor of } \mathrm{CTn} 1 \text {, } \\
\text { erm }(\mathrm{B}) \text { present in } \mathrm{CD} 0386 \text { within } \mathrm{CTn} 1\end{array}$ & 9 \\
\hline $\begin{array}{l}630 \Delta \text { erm } \\
\text { tcdB::erm(B) (012) }\end{array}$ & $\begin{array}{l}\text { ClosTron mutant, contains erm(B) } \\
\text { inserted within } t c d B\end{array}$ & 4 \\
\hline OX904 (138) & Non-toxigenic recent clinical isolate & 13 \\
\hline OX2157 (140) & Non-toxigenic recent clinical isolate & 13 \\
\hline OX904rif (138) & $\begin{array}{l}\text { Rifampicin-resistant derivative of } \\
\text { OX904 }\end{array}$ & This study \\
\hline OX2157rif (140) & $\begin{array}{l}\text { Rifampicin-resistant derivative of } \\
\text { OX2157 }\end{array}$ & This study \\
\hline PaLoc386 & $\begin{array}{l}\text { CD37 transconjugant containing } \\
\text { the } 630 \Delta \text { erm wild-type PaLoc }\end{array}$ & This study \\
\hline PaLoc12 & $\begin{array}{l}\text { CD37 transconjugant containing } \\
\text { the ClosTron tcdB::erm(B) PaLoc }\end{array}$ & This study \\
\hline PaLoc22 & $\begin{array}{l}\text { CD37 transconjugant containing } \\
\text { the ClosTron tcdB::erm(B) PaLoc }\end{array}$ & This study \\
\hline PaLoc26 & $\begin{array}{l}\text { CD37 transconjugant containing } \\
\text { the ClosTron tcdB::erm(B) PaLoc }\end{array}$ & This study \\
\hline PaLoc35 & $\begin{array}{l}\text { CD37 transconjugant containing } \\
\text { the ClosTron tcdB::erm(B) PaLoc }\end{array}$ & This study \\
\hline PaLoc37 & $\begin{array}{l}\text { CD37 transconjugant containing } \\
\text { the ClosTron tcdB::erm(B) PaLoc }\end{array}$ & This study \\
\hline PaLoc46 & $\begin{array}{l}\text { CD37 transconjugant containing } \\
\text { the ClosTron tcdB::erm(B) PaLoc }\end{array}$ & This study \\
\hline
\end{tabular}

the PaLoc in strain PaLoc386 was identical to that of the donor. In the other transconjugants, the PaLoc contained the clostron insertion, but was otherwise identical to the PaLoc in $630 \Delta \mathrm{erm}$. To define the horizontally transferred region in the transconjugants, it was necessary to distinguish between donor and recipient genome. To do this, 30 regions of $\sim 10 \mathrm{~kb}$ evenly spaced around the $630 \Delta \mathrm{erm}$ genome were selected (shown schematically in Fig. 2 as green bars with the sequences given in SI1) and compared with the corresponding regions of the CD37 genome ${ }^{12}$. These regions were chosen as they did not contain any predicted mobile genetic elements such as CTns, IStrons or prophages. The presence of 10-150 single-nucleotide polymorphisms (SNPs) and indels in these 30 regions allowed discrimination between the donor and recipient DNA. Alignment of the genomic DNA sequence of the PaLoc-containing transconjugants in the 30 regions showed that in all cases, regions $1-3$ and 8-30 were derived from the recipient. In the donor strains, $630 \Delta \mathrm{erm}$ and $630 \Delta \mathrm{erm} \mathrm{tcdB}: \mathrm{erm}(\mathrm{B})$, and in all of the transconjugants, the PaLoc is located between regions 6 and 7. In the transconjugants, the PaLoc has transferred on variable-sized DNA fragments that include either region 6 or 7 from the donor strain in 6 out of 7 transconjugants, and regions 5 and 6 from the donor in transconjugant PaLoc385. In transconjugant PaLoc26, the PaLoc was transferred from the donor on a relatively small DNA fragment (a maximum size of $67,644 \mathrm{bp}$ ) and both regions 6 and 7 in this transconjugant are derived from the recipient. In transconjugants, PaLoc386 and PaLoc37, Tn5397 and the PaLoc have transferred on the same DNA fragment (Fig. 2). This contrasts with the other cotransfers where the CTn and the PaLoc transferred on separate DNA fragments. For example, in transconjugant PaLoc386, CTn5 also transferred but 
a

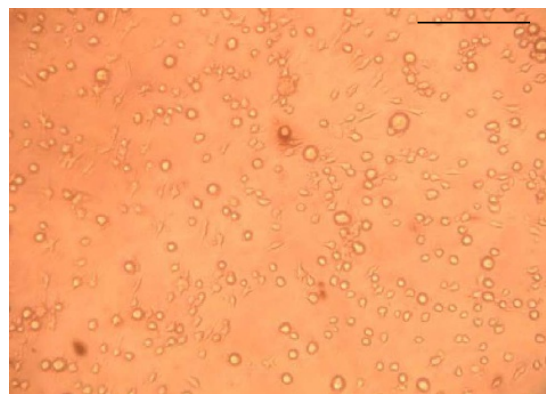

b

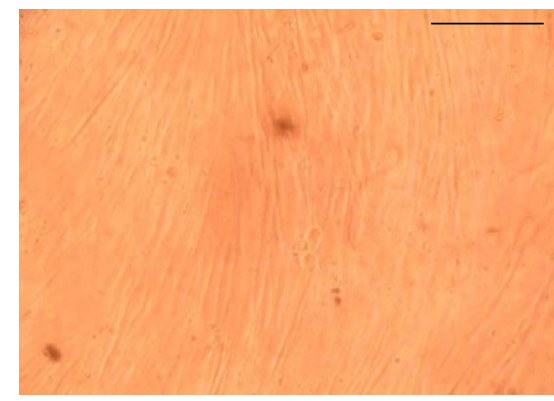

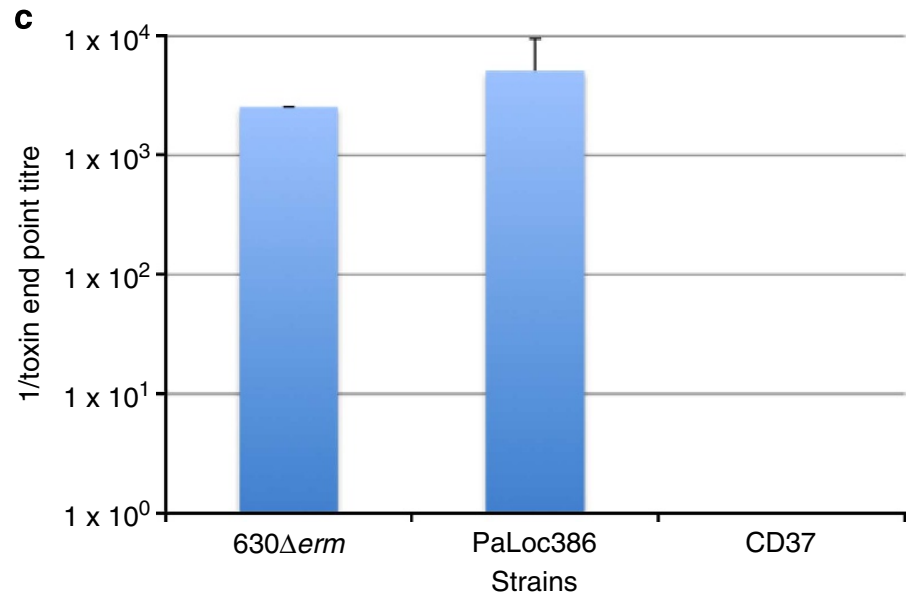

Figure 1 | Cytotoxicity assay comparing toxin production. Toxin production by the donor, transconjugant and recipient strains were compared using a cytotoxicity assay. (a) HFF-1 cells incubated with the culture supernatant of transconjugant PaLoc386 show rounding of the cells. (b) The cytotoxic effect of PaLoc386 can be neutralized using antibodies against TcdB. Scale bar, $1 \mathrm{~mm}$ in $\mathbf{a}$ and $\mathbf{b}$. (c) The toxin end point titre was determined for culture supernatants of donor strain 630 Derm, transconjugants PaLoc386 and the recipient CD37. The mean \pm s.d. is shown for three independent experiments.

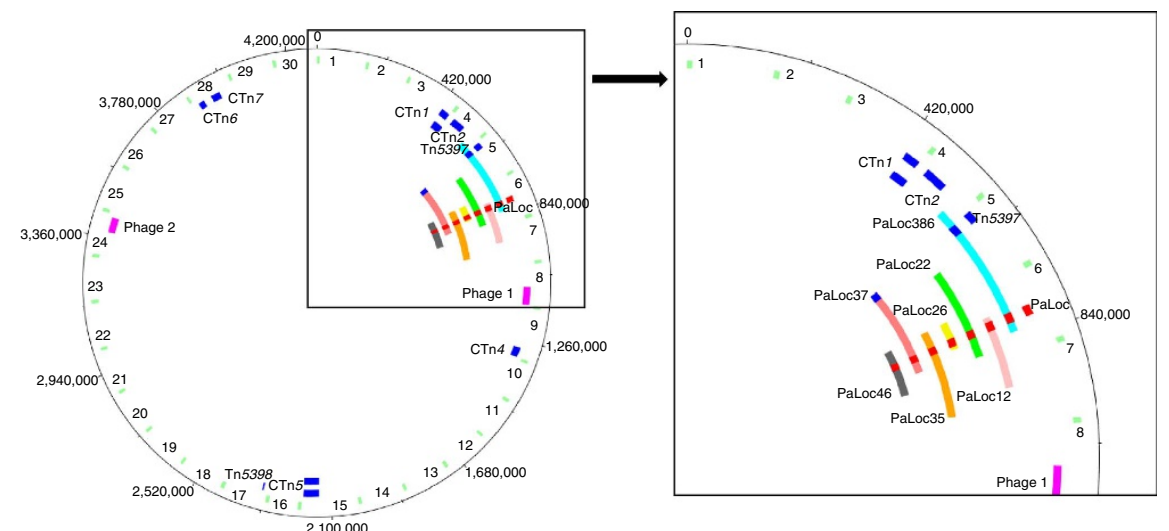

Figure 2 | Schematic representation of the donor-specific DNA fragments present in the transconjugants. The circular chromosome of $C$. difficile strain 630 is shown. The boxed region on the left is magnified on the right. The green fragments in the outer ring represent the $10 \mathrm{~kb}$ fragments used to differentiate transconjugants from donors; see text for more details. The dark blue fragments in the second ring indicate the mobile genetic elements present in the donor; the red fragment in this ring represents the PaLoc. The donor-specific fragments that are present in the transconjugants are shown in the seven inner-most rings as coloured fragments with the number of each transconjugant indicated. The red fragments in these represent the transferred PaLoc. The dark blue fragments in the track of PaLoc386 depict CTn1 and CTn5 that are both present in this transconjugant.

independently of the PaLoc and has inserted near region 16 in the transconjugant (Fig. 2). In contrast to PaLoc transfer, in which the flanking DNA was also derived from the donor, there was no evidence for the transfer of flanking DNA in the case of CTn5. The remaining PaLoc-containing transconjugants shown in Fig. 2 did not contain CTn2, CTn4, CTn5, CTn7 or $\operatorname{Tn} 5397$.
The approximate length of the transferred DNA in each of the transconjugants was determined by calculating the distance from the first SNP or indel upstream of the PaLoc to the last SNP or indel downstream of the PaLoc that was specific to the donor strain $630 \Delta \mathrm{erm}$. Because of the high level of sequence identity between CD37 and $630 \Delta$ erm in the regions flanking the PaLoc, this analysis could only determine the maximum and minimum 


\begin{tabular}{|lcc|}
$\begin{array}{l}\text { Table } 2 \\
\text { strain. }\end{array}$ & Size of the DNA fragments inserted in the recipient \\
Isolate & $\begin{array}{c}\text { Minimum size of DNA } \\
\text { fragment transferred }\end{array}$ & $\begin{array}{c}\text { Maximum size of DNA } \\
\text { fragment transferred }\end{array}$ \\
\hline PaLoc386 & $271,992 \mathrm{bp}$ & $272,977 \mathrm{bp}$ \\
PaLoc12 & $151,692 \mathrm{bp}$ & $155,024 \mathrm{bp}$ \\
PaLoc22 & $157,688 \mathrm{bp}$ & $170,386 \mathrm{bp}$ \\
PaLoc26 & $66,034 \mathrm{bp}$ & $67,644 \mathrm{bp}$ \\
PaLoc35 & $224,973 \mathrm{bp}$ & $228,497 \mathrm{bp}$ \\
PaLoc37 & $250,027 \mathrm{bp}$ & $252,009 \mathrm{bp}$ \\
PaLoc46 & $141,658 \mathrm{bp}$ & $145,290 \mathrm{bp}$ \\
\hline
\end{tabular}

size of the transferred fragment (Table 2, sequences included in SI2). The PaLoc was transferred on variable-sized DNA fragments ranging from at least $66,034 \mathrm{bp}$ to a maximum of 272,977 bp (Table 2 and Fig. 2).

To exclude transformation as a mechanism of transfer, the mating experiments were repeated in the presence of DNase and no difference in the frequency of transfer was detected. It was also possible that the PaLoc could be transferred by transduction by either of the prophages that are present in strain $630 \Delta \mathrm{erm}$ (Fig. 2). We think this is improbable given that the transferred DNA is much larger than the phage genomes and is unlikely therefore to be packaged within the phage heads. Nonetheless concentrated phage suspensions were made by mitomycin $C$ induction of $630 \Delta \mathrm{erm} \mathrm{tcdB}: \mathrm{erm}(\mathrm{B})$. We attempted to infect strain CD37 with these phage suspensions but did not obtain plaques, indicating that this strain is resistant to these phages. Strain CD843 was used to confirm the presence of infectious phages in the suspensions. Although plaques were produced with this host, no lincomycin-resistant transductants were obtained in this phage assay. These results indicate that PaLoc transfer is unlikely to occur via transduction.

The PaLoc can transfer into other non-toxigenic strains. Recent work by Dingle et al. ${ }^{13}$, analysing the molecular epidemiology of a large population of $C$. difficile strains, has suggested that PaLoc transfer is occurring in wild populations. We tested two of the non-toxigenic isolates from this study (OX904 belonging to clade 4, PCR-ribotype 138 and OX2157 belonging to clade 1, PCRribotype 140) to determine whether they could act as recipients for the marked PaLoc in matings using 630 $\Delta \mathrm{erm} \mathrm{tcdB}:: \mathrm{erm}(\mathrm{B})$ as donor. To do this, spontaneous rifampicin-resistant derivatives of these strains were first isolated (see Methods and Table 1). The PaLoc was transferred to OX2157rif at a frequency of $4.4 \times 10^{-8}$ and to OX904rif at a frequency of $2.3 \times 10^{-9}$, indicating that PaLoc transfer is not restricted to particular donor and recipient pairs.

\section{Discussion}

This work demonstrates that the PaLoc of $C$. difficile 630 4 erm is capable of transfer by a conjugation-like mechanism to nontoxigenic strains. In the case of $C$. difficile CD37, we showed that this resulted in its conversion to a toxin producer. This finding has important clinical implications, as non-toxigenic strains have shown promise as a treatment for $\mathrm{CDAD}^{14}$. Clearly, if the PaLoc is capable of transfer to strains being used for treatment, then we urgently need to understand the conditions under which transfer of the PaLoc is selected. Non-toxigenic strains are found coexisting with toxigenic ones ${ }^{13}$, providing opportunity for the PaLoc to spread within the $C$. difficile populations. Also of concern is the finding that transfer of the PaLoc often, but not always, occurs with cotransfer of conjugative transposons encoding resistance to antibiotics, resulting in the cotransfer of virulence and antibiotic-resistance genes. This is illustrated in the transconjugants PaLoc386 and PaLoc37, in which Tn5397 (encoding tetracycline resistance) and the PaLoc were transferred on the same DNA fragment from $630 \Delta e r m$ to CD37. CTns and the PaLoc can also be transferred on separate DNA fragments as shown by transconjugant PaLoc 386 in which CTn 5 and CTn 1 are transferred and integrated into their preferred genomic sites $^{9}$ (between regions 15 and 16 for CTn5, and between regions 3 and 4 for CTn1 (Fig. 2)) with no detectable transfer of flanking DNA. Presumably in this case, CTn5 and CTn1 transferred by excision to form a circular molecule that was nicked at the origin of transfer (oriT) and transferred as a distinct genetic element.

A striking observation is that although transfer of the PaLoc and the CTns occurs at a low frequency (between $10^{-7}$ and $10^{-9}$ transconjugants per donor), cotransfer of unselected genetic elements (including the PaLoc) is observed. A probable explanation for this is that when one or more of the CTns initiates a transfer event, other elements present in the same cell are activated by trans-acting regulatory molecules. This type of interaction has been observed between Tn916-like elements ${ }^{15}$. The first step in transfer by conjugative and mobilizable transposons is excision to form a circular molecule that is nicked at the oriT, and a single strand is transferred to a suitable recipient via a mating pore in a complex process analogous to type IV secretion ${ }^{16}$. However, the PaLoc is not contained within an obvious mobile element and is transferred on DNA fragments of variable size in different transconjugants. This type of transfer is reminiscent of that mediated by high-frequency recombination (Hfr) in which an oriT within the chromosome can mediate transfer of the chromosome ${ }^{17}$. In the recipient, the incoming chromosomal DNA is integrated into the chromosome by homologous recombination. In theory, the whole chromosome can be transferred, although this rarely happens due to disruption of the mating pair or nicking of the incoming DNA strand, so that markers proximal to the integrated oriT are transferred at a higher frequency than those located distally. There are examples of CTns mediating Hfr-type transfer, for example, in Vibrio cholerae $^{18}$ and Bacteroides sp. ${ }^{19}$ In these cases, the CTns do not excise so that instead of just mobilizing themselves, the chromosome is also mobilized. Three transfer-proficient CTns in the C. difficile genome, CTn1, CTn2 and Tn5397 are close to the PaLoc (Fig. 2) and are candidates for mediation of $\mathrm{Hfr}$ transfer. The regions flanking the PaLoc in 630 $\mathrm{erm}$ are homologous to chromosomal DNA of the non-toxigenic strain, CD37, and, consequently, the incoming DNA from the donor could recombine with the recipient chromosome resulting in integration of this region. Whole-genome sequence comparison has indicated that large blocks of DNA have transferred between C. difficile strains in the past, indicating that this form of transfer is an important driver of $C$. difficile genome evolution ${ }^{20}$.

A recent study of diversity in the $C$. difficile S-layer cassette suggests that this cassette is undergoing horizontal gene transfer across genotypes ${ }^{21}$. The recombination events for the cassette shown in this study were significantly smaller $(12-35 \mathrm{~kb})$ than those reported here for the PaLoc, and the recombining loci are located $\sim 2 \mathrm{Mb}$ apart on the chromosome. Although transfer of the S-layer cassette has not been demonstrated, it is possible that this region can transfer by a mechanism similar to that of the PaLoc, a hypothesis that requires investigation.

\section{Methods}

Strains and growth conditions. All strains used in this study are listed in SI 3. C. difficile strains were grown in brain-heart infusion (BHI) broth or on BHI agar 
(Oxoid Ltd, Basingstoke, UK) supplemented with 5\% defibrinated horse blood (E \& O laboratories, Bonnybridge, UK). For the cytotoxicity assay, C. difficile was grown in tryptone, yeast (TY) broth consisting of 3\% Bacto Tryptose (BD Biosciences, Oxford, UK), $2 \%$ yeast extract (Oxoid Ltd) and $0.1 \%$ thioglycolate (Sigma-Aldrich Company Ltd, UK) at $\mathrm{pH}$ 7.4. C. difficile strains were grown at $37^{\circ} \mathrm{C}$ under anaerobic conditions $\left(80 \% \mathrm{~N}_{2}, 10 \% \mathrm{H}_{2}\right.$ and $\left.10 \% \mathrm{CO}_{2}\right)$.

Molecular biology techniques. DNA was isolated using the Gentra Puregene Yeast/Bact. Kit (Qiagen, UK). PCR amplification was carried out using the NEB Taq polymerase kit (New England Biolabs, UK) according to the manufacturer's instructions.

Filter-matings. Donor and recipient strains were grown in $\mathrm{BHI}$ broth to an $\mathrm{OD}_{600}$ of $\sim 0.5-0.6$. Cells were washed, pellets were taken up into BHI broth and donor and recipient cultures were mixed. The cell mixture was spread onto $0.45 \mu \mathrm{m}$ pore size cellulose nitrate filters (Sartorius, Epsom, UK) on BHI agar plates. In some cases DNase was added to the mating plates at a concentration of $50 \mu \mathrm{g} \mathrm{ml}^{-1}$. After $24 \mathrm{~h}$, the filters were placed in $25 \mathrm{ml}$ tubes containing $1 \mathrm{ml}$ BHI broth and were vortexed vigorously. The cell mixture was spread onto selective plates to determine donor, recipient and transconjugant cell numbers on the filters.

Putative transconjugants were assessed by $\mathrm{PCR}$ for the presence of the erm $(\mathrm{B})$ selection marker using primers ErmRAM-F (5'-ACGCGTTATATTGATAAAAA TAATAATAGTGGG- $3^{\prime}$ ) and ErmRAM-R (5'-ACGCGTGCGACTCATAGAATT ATTTCCTCCCG- $\left.3^{\prime}\right)^{22}$. The presence of the PaLoc was determined by PCR using primers tcdA-F (5'-CATCTATAAGTTCTCATATTCCTTC- $\left.{ }^{\prime}\right)$ and tcdA-R (5'-CCAGAAACTTATATTGTCC- $\left.{ }^{\prime}\right)$. The absence of the CDT locus was determined using primers cdtA-F (5' $5^{\prime}$-(C/T)AATACTACTTACAAGGCTCC- $\left.3^{\prime}\right)$ and cdtA-R ( $5^{\prime}$-TTTCGTTTTGATTTT(C/T)TGTTC-3'). Spontaneous rifampicinresistant mutants were isolated by plating $10^{10}$ bacteria on agar plates containing $25 \mu \mathrm{g} \mathrm{ml}^{-1}$ rifampicin. As the complete genome sequence of strains OX904 and OX2157 is not available, transconjugants were differentiated from spontaneous rifampicin-resistant mutants of the donor by PCR and sequencing of genes cwp66 (primers 5'AAACCWAHARYYAATGATACAG and 5'RTWGWTYTTCCCCAT CKWGAAAC) and gene CD25780 (primers 5'GATGATATAGACTTTACATC AGAAG and 5'CAGCCGCAATTACTTTTAATC) encoding a predicted serine threonine protein kinase.

Cytotoxicity assay. HFF-1 cells were grown in Dulbecco's Modified Eagle Medium (DMEM) (Life technologies) supplemented with $10 \%$ heat-inactivated fetal calf serum, $2 \mathrm{mM}$ glutamine, 100 units ml $^{-1}$ penicillin and $100 \mu \mathrm{g} \mathrm{ml}^{-1}$ streptomycin at $37^{\circ} \mathrm{C}$ in $5 \% \mathrm{CO}_{2}$. Cells were subcultured by washing twice with sterile PBS and incubating with $0.1 \%$ trypsin for $5 \mathrm{~min}$. For the cytotoxicity assay, the cells were seeded at $10^{4}$ cells per well in 96-well plates and incubated for $24 \mathrm{~h}$ in $5 \% \mathrm{CO}_{2}$.

Overnight $C$. difficile cultures were diluted 1:10 into tryptone, yeast (TY) broth and incubated without shaking for $48 \mathrm{~h}$. The cultures were centrifuged for $10 \mathrm{~min}$ at $4,500 \mathrm{~g}$ and the supernatant was filter-sterilized. A dilution series of the supernatant was prepared in PBS. Each dilution was added to confluent HFF-1 cells in duplicate. After $24 \mathrm{~h}$, the cells were assessed for CPE using an inverted microscope. The toxin end point titre was recorded as the lowest dilution in the series where the cells were indistinguishable from the negative control. C. difficile antitoxin $(C$. difficile test kit, T5003Techlab Inc, Blacksburg, VA, USA) was added to one of the duplicate wells to determine whether the CPE was neutralized.

Genomic sequencing. Genomic sequencing was performed by UCL Genomics as part of a 72-bp paired end run, performed on an Illumina GAII-X. Genome assembly was performed using the Velvet software suite ${ }^{23}$ and reads were mapped against strain 630 (AM180355) using $\mathrm{XBase}^{24}$. The transconjugant sequences were compared with the sequence of strain 630 (AM180355) and the shotgun sequence of strain CD37 (AHJJ01000000) using the Artemis Comparison Tool ${ }^{25}$. SNP analysis was performed by alignment using eBioX version 1.51 . Sequence of the chromosomal DNA used to compare the donor- and recipient-specific DNA in the transconjugants is provided in Supplementary Data 1. Sequence at the junctions between the chromosome and the donor-specific fragment is provided in Supplementary Data 2. The ends on either side of the transferred fragment are compared with the donor and recipient sequences.

\section{References}

1. Rupnik, M., Wilcox, M. H. \& Gerding, D. N. Clostridium difficile infection: new developments in epidemiology and pathogenesis. Nat. Rev. Microbiol. 7, 526-536 (2009).

2. Bartlett, J. G. Historical perspectives on studies of Clostridium difficile and C. difficile infection. Clin. Infect. Dis. 46(Suppl 1): S4-11 (2008).

3. Lyras, D. et al. Toxin B is essential for virulence of Clostridium difficile. Nature 458, 1176-1179 (2009).

4. Kuehne, S. A. et al. The role of toxin A and toxin B in Clostridium difficile infection. Nature 467, 711-713 (2010).
5. Braun, V., Hundsberger, T., Leukel, P., Sauerborn, M. \& von Eichel-Streiber, C. Definition of the single integration site of the pathogenicity locus in Clostridium difficile. Gene 181, 29-38 (1996).

6. Hussain, H. A., Roberts, A. P. \& Mullany, P. Generation of an erythromycinsensitive derivative of Clostridium difficile strain 630 (630Deltaerm) and demonstration that the conjugative transposon Tn916DeltaE enters the genome of this strain at multiple sites. J. Med. Microb. 54, 137-141 (2005).

7. Sebaihia, M. et al. The multidrug-resistant human pathogen Clostridium difficile has a highly mobile, mosaic genome. Nat. Genet. 38, 779-786 (2006).

8. Roberts, A. P. \& Mullany, P. Tn916-like genetic elements: a diverse group of modular mobile elements conferring antibiotic resistance. FEMS Microbiol. Rev. 35, 856-871 (2011).

9. Brouwer, M. S., Warburton, P. J., Roberts, A. P., Mullany, P. \& Allan, E. Genetic organisation, mobility and predicted functions of genes on integrated, mobile genetic elements in sequenced strains of Clostridium difficile. PLoS One 6, e23014 (2011)

10. Mullany, P. et al. Genetic analysis of a tetracycline resistance element from Clostridium difficile and its conjugal transfer to and from Bacillus subtilis. J. Gen. Microbiol. 136, 1343-1349 (1990).

11. Wang, H. et al. Characterization of the ends and target sites of the novel conjugative transposon Tn5397 from Clostridium difficile: excision and circularization is mediated by the large resolvase, TndX. J. Bacteriol. 182, 3775-3783 (2000).

12. Brouwer, M. S., Allan, E., Mullany, P. \& Roberts, A. P. Draft genome sequence of the nontoxigenic Clostridium difficile strain CD37. J. Bacteriol. 194, 2125-2126 (2012).

13. Dingle, K. E. et al. Clinical Clostridium difficile: clonality and pathogenicity locus diversity. PLoS One 6, e19993 (2011).

14. Villano, S. A., Seiberling, M., Tatarowicz, W., Monnot-Chase, E. \& Gerding, D. N. Evaluation of an oral suspension of VP20621, spores of nontoxigenic Clostridium difficile strain M3, in healthy subjects. Antimicrob. Agents Chemother. 56, 5224-5229 (2012).

15. Flannagan, S. E. \& Clewell, D. B. Conjugative transfer of Tn916 in Enterococcus faecalis: trans activation of homologous transposons. J. Bacteriol. 173, 7136-7141 (1991).

16. Juhas, M., Crook, D. W. \& Hood, D. W. Type IV secretion systems: tools of bacterial horizontal gene transfer and virulence. Cell. Microbiol. 10, 2377-2386 (2008).

17. Kahn, P. L. Isolation of high-frequency recombining strains from Escherichia coli containing the V colicinogenic factor. J. Bacteriol. 96, 205-214 (1968).

18. Hochhut, B., Marrero, J. \& Waldor, M. K. Mobilization of plasmids and chromosomal DNA mediated by the SXT element, a constin found in Vibrio cholerae O139. J. Bacteriol. 182, 2043-2047 (2000).

19. Whittle, G., Hamburger, N., Shoemaker, N. B. \& Salyers, A. A. A bacteroides conjugative transposon, CTnERL, can transfer a portion of itself by conjugation without excising from the chromosome. J. Bacteriol. 188, 1169-1174 (2006).

20. He, M. et al. Evolutionary dynamics of Clostridium difficile over short and long time scales. Proc. Natl Acad. Sci. 107, 7525-7532 (2010).

21. Dingle, K. E. et al. Recombinational switching of the Clostridium difficile S-layer and a novel glycosylation gene cluster revealed by large-scale whole-genome sequencing. J. Infect. Dis. 207, 675-686 (2013).

22. Heap, J. T., Pennington, O. J., Cartman, S. T., Carter, G. P. \& Minton, N. P. The ClosTron: a universal gene knock-out system for the genus Clostridium. J. Microbiol. Methods 70, 452-464 (2007)

23. Zerbino, D. R. \& Birney, E. Velvet: algorithms for de novo short read assembly using de Bruijn graphs. Genome Res. 18, 821-829 (2008).

24. Chaudhuri, R. R. et al. xBASE2: a comprehensive resource for comparative bacterial genomics. Nucleic. Acids Res. 36, D543-D546 (2008).

25. Carver, T. et al. Artemis and ACT: viewing, annotating and comparing sequences stored in a relational database. Bioinformatics. 24, 2672-2676 (2008).

26. Wüst, J. \& Hardegger, U. Transferable resistance to clindamycin, erythromycin, and tetracycline in Clostridium difficile. Antimicrob. Agents Chemother. 23, 784-786 (1983).

27. Smith, C. J., Markowitz, S. M. \& Macrina, F. L. Transferable tetracycline resistance in Clostridium difficile. Antimicrob. Agents Chemother. 19, 997-1003 (1981).

28. Goh, S., Ong, P. F., Song, K. P., Riley, T. V. \& Chang, B. J. The complete genome sequence of Clostridium difficile phage phi 2 and comparisons to phiCD119 and inducible prophages of CD630. Microbiology. 153, 676-685 (2007).

\section{Acknowledgements}

We thank Dr Sarah Kuehne, Dr Shan Goh and Dr Kate Dingle for providing the bacterial strains. We would like to thank the sequencing team at UCL Genomics for performing the genomic sequencing and for their help with the analysis of genomic sequences. The research leading to these results has received funding from the European Community's Seventh Framework Programme (FP7/2007-2013) under grant agreement no. 223585 and the Medical Research Council (grant no. G0601176). 


\section{Authors contributions}

M.S.M.B., P.M., E.A. and A.P.R. designed the experiments and wrote the manuscript M.S.M.B., R.W. and H.H. carried out the experiments.

\section{Additional information}

Supplementary Information accompanies this paper at http://www.nature.com/

naturecommunications

Competing financial interests: The authors declare no competing financial interests.
Reprints and permission information is available online at http://npg.nature.com/ reprintsandpermissions/

How to cite this article: Brouwer, M. S. M. et al. Horizontal gene transfer converts nontoxigenic Clostridium difficile strains into toxin producers. Nat. Commun. 4:2601 doi: 10.1038/ncomms3601 (2013).

(c) (i) This article is licensed under a Creative Commons Attribution 3.0 Unported Licence. To view a copy of this licence visit http:// creativecommons.org/licenses/by/3.0/. 